\title{
Hepatitis B virus core protein with hot-spot mutations inhibit MxA gene transcription but has no effect on inhibition of virus replication by interferon $\alpha$
}

\author{
Yu Zhijian ${ }^{1}$, Huang Zhen ${ }^{1}$, Zhang Fan², Yang Jin ${ }^{1}$, Deng Qiwen ${ }^{1 *}$, Zeng Zhongming ${ }^{1 *}$
}

\begin{abstract}
It has been reported that hepatitis B virus (HBV) core protein $(\mathrm{HBC})$ can inhibit the transcription of human interferon-induced MXA gene. In this study, we investigated whether HBc protein mutations at hot spots (L60V, S87G and 197L) could still inhibit MxA transcription and the potential significance of this inhibition in virus replication in vitro. Our data indicated that the IFN-induced MxA mRNA expression level and MxA promoter activity was significantly down-regulated by mutant protein of $\mathrm{HBC}(197 \mathrm{~L})$, compared to WT and the other two mutated HBC proteins(L60V or S87G). However, in Huh7 cells stably expressing WT or the mutated HBc proteins (L60V, S87G or 197L), IFN- $\alpha$ could inhibit the extra- and intracellular HBV DNA level and HBsAg secretion to a similar level compared to that in cells transfected with control plasmids. In conclusion, HBc protein with 197L mutation may play an especial role in suppressing the transcription of MxA gene. Moreover, the inhibitory effect on MxA gene transcription by the WT or mutated HBc proteins (L60V, S87G and 197L) has no impact on inhibition of HBV replication by IFN- $\alpha$ in Huh7 cells. The clinical significance of the inhibitory effect of MxA gene transcription by HBc protein requires further study.
\end{abstract}

\section{Introduction}

Many studies have indicated that hepatitis B virus (HBV) core gene mutations are significantly associated with hepatitis activity in patients with chronic hepatitis $\mathrm{B}(\mathrm{CHB})[1-4]$. In addition, gene mutations in the precore/core region of HBV occur more frequently in patients with severe or fulminant hepatitis compared to asymptomatic carriers and those with acute self-limited hepatitis [1-3]. Several investigations have shown that the substitutions L60V, S87G and I97L in the HBV core antigen ( $\mathrm{HBcAg}$, referred to as the $\mathrm{HBc}$ protein) were the most frequent in patients with $\mathrm{CHB}$, and $\mathrm{HBV}$ with these "hot-spot" mutations show different characteristics in replication cycle in vitro compared to the wild-type strain [4-9]. Moreover, in vivo infection with full-length HBV strains carrying these hot-spot mutations could

\footnotetext{
* Correspondence: qiwendeng@hotmail.com; zmzeng@vip.163.com 'Department of Infectious Diseases, the Affiliated Shenzhen Nanshan Hospital of Guangdong Medical College, Shenzhen, China Full list of author information is available at the end of the article
}

alter the immune recognition sites of $\mathrm{HBc}$ protein thereby eliciting or evading immune clearance [4]. Recently, multiple reports have demonstrated that $\mathrm{HBc}$ protein can have various effects on expression and transcription of some intracellular cytokines and proteins [10-12]. However, it is unknown whether HBc protein with hot-spot mutations would play a different role compared to the wild-type (WT).

Intracellular transcription and expression of human MxA protein is specifically dependent on induction by type I interferon (IFN), and furthermore, MxA protein plays an important antiviral role as a downstream mediator of type I interferon [13-15]. Human MxA protein, a GTPase, can inhibit the replication of a wide range of negative- and positive-strand RNA viruses as well as HBV $[14,16,17]$. Recently, HBc protein has been shown to trans-suppress IFN-induced gene expression and to down-regulate the promoter activity of the MxA gene by direct interaction with the IFN-stimulated response element (ISRE) sequence of the MxA promoter [12,18]. 
However, it is still unknown whether HBc protein carrying the hot-spot mutations has a different effect on transcription and expression of the MxA gene compared to the wild-type (WT). Furthermore, it remains to be elucidated whether the inhibition of MxA gene transcription by $\mathrm{HBc}$ protein influences the inhibition of HBV replication by IFN- $\alpha$.

\section{Materials and methods}

\section{Plasmid constructs}

The parental plasmid for pU19-1.24HBV was kindly provided by Dr. Mizokami [14]. The WT HBc gene was amplified from pU19-1.24HBV using the sense primer: 5'-GGGGCCTAAAA CTCAGACAACTATTG-3' and antisense primer: 5'-GCAAGCTATTGTGTGTTGG-3'. pCMV-HBc (WT), the expression vector for WT HBc protein, was constructed with the WT HBc gene inserted into pCMV-Tag1 (containing the Flag-tag, purchased from Stratagene Company) by standard procedures. Using pCMV-HBc (WT), the other three plasmids [pCMV-HBc (L60V), pCMV-HBc (S87G) and pCMV-HBc (I97L)] expressing HBc proteins with the substitutions L60V, I97L and S87G, respectively, were constructed using the Quick Change Site-Directed Mutagenesis Kit (Stratagene, USA) and the primers described previously [4]. pCMV-HBc (WT), pCMV-HBc (L60V), pCMV-HBc (S87G) and pCMV-HBc (I97L) were confirmed by sequencing and these vectors were able to express the $\mathrm{HBc}$ protein/Flag-tag fused protein. The pMxA550-Luc plasmid was constructed by insertion of the $550 \mathrm{bp}$ minimal MxA gene promoter $(+553$, -10) in front of the luciferase gene of the pGL3 basic vector (Promega) as described previously [19]. Renilla luciferase vector was purchased from Promega.

\section{Cell culture, transfection, harvest and measurement of Luciferase activity}

First, the influence of the $\mathrm{HBc}$ proteins (WT and mutated) on the expression level of MxA mRNA in Huh7 cells was determined. Huh7 cells $\left(2 \times 10^{5}\right.$ per well) were seeded in 12-well plates, with $0.5 \mathrm{~mL}$ DMEM media containing 10\% FBS per well (Gibco). After $24 \mathrm{~h}$, Huh7 cells were transfected with $0.5 \mu \mathrm{g}$ pCMV-HBc (WT), pCMV-HBc (L60V), pCMV-HBc (S87G), pCMVHBc (I97L) and control DNA (pCMV-Tag1 and salmon DNA), respectively, using FuGene HD transfection reagent (Roche). After transfection for $48 \mathrm{~h}$, culture medium was removed and fresh media with IFN- $\alpha$ $(1000 \mathrm{IU} / \mathrm{mL})$ was added and incubated for a further 8 $\mathrm{h}$ at $37^{\circ} \mathrm{C}$. Cells were then collected for detection of $\mathrm{HBc}$ protein expression by western blot as described below, and used for extraction of total RNA by Trizol (Invitrogen). Levels of MxA mRNA were assessed by real-time PCR as described below. In addition, the influence of the $\mathrm{HBc}$ proteins (WT and mutated) on the promoter activity of the MxA gene was examined. Huh7 cells were seeded in 12-well plates at $2 \times 10^{5}$ cells per well and, after $24 \mathrm{~h}$, pMxA550-Luc $(0.25 \mu \mathrm{g})$ was cotransfected in Huh7 cells with $0.5 \mu \mathrm{g}$ pCMV-HBc (WT), pCMV-HBc (L60V), pCMV-HBc (S87G), pCMV-HBc (I97L) and control DNA (pCMV-Tag1 and salmon DNA), respectively, using FuGene HD (Roche). After $48 \mathrm{~h}$, the culture medium was removed and fresh media with IFN- $\alpha(1000 \mathrm{IU} / \mathrm{mL})$ was added and incubated for a further $8 \mathrm{~h}$ at $37^{\circ} \mathrm{C}$. The cells were then lysed and collected and the luciferase activity in the cellular lysate was measured by Glomax (Promega) according to the manual of the Dual-Luciferase Reporter Assay System (Promega). Renilla luciferase vector (40 ng) was used to control the transfection efficiency.

Moreover, we wished to examine whether the inhibition of MxA transcription by $\mathrm{HBc}$ protein would decrease the inhibitory effect of IFN- $\alpha$ on HBV replication. pCMV-HBc (WT), pCMV-HBc (L60V), pCMVHBc (S87G), pCMV-HBc (I97L) and pCMV-Tag1 were transfected into Huh7 cells as described above, using 0.5 $\mu \mathrm{g}$ of DNA. Subsequently, transfected cells were selected with neomycin $(100 \mathrm{mg} / \mathrm{mL})$. After 2 weeks, the positive cells transfected with the above vectors were identified to express WT or the mutated HBc protein by western blot as described below. The cells stably expressing WT or mutated $\mathrm{HBC}$ protein, and control cells transfected with the empty vector (pCMV-Tag1), were seeded in 10 $\mathrm{cm}$ dishes respectively. After $24 \mathrm{~h}$, pU19-1.24HBV (10 $\mu \mathrm{g}$ ) and Renilla luciferase vector (60 ng) were co-transfected into the Huh7 cells. After transfection for $24 \mathrm{~h}$, culture medium was removed and fresh media with IFN- $\alpha(1000 \mathrm{IU} / \mathrm{mL})$ was added and incubation continued for a further $24 \mathrm{~h}$ at $37^{\circ} \mathrm{C}$. Then, the relative $\mathrm{MxA}$ and GAPDH mRNA levels, HBV DNA and hepatitis B surface antigen ( $\mathrm{HBsAg}$ ) were assessed as described below.

\section{Western blot analysis}

For SDS-PAGE, cells were lysed in lysis buffer (Cell Signaling Technologies), and the protein content measured using the Bradford technique (Bio-Rad). Twenty-one micrograms of total protein was boiled in SDS loading buffer and loaded onto the gel. Proteins were then transferred to a nitrocellulose membrane (Amersham). After incubation with blocking solution (Invitrogen), the blots were incubated with the antibody (anti-Flag, 1:3000; poly-anti-rabbit $\beta$-actin, 1:500). The monoclonal anti-Flag antibody was purchased from the Invitrogen Company and poly-anti-rabbit $\beta$-actin antibody was purchased from the Santa Cruz Biotechnology. Detection was carried out using a horseradish peroxidase-conjugated secondary antibody (1:2000) (Santa Cruz 
Biotechnology) and chemi-luminescence development was carried out (Pierce).

\section{Real-time analysis of MxA mRNA and HBV DNA levels} For real-time analysis of MxA mRNA detection, $500 \mathrm{ng}$ of total RNA were converted to cDNA using TaqMan reverse transcription reagents (Applied Biosystems, Foster City, CA, USA). Real-time PCR to detect MxA mRNA and GAPDH mRNA levels was performed with the reported methods, DNA primers and probes[20]. Relative amount of MxA mRNA level were reported as number of folds of MxA mRNA copies relative to the expression of GAPDH. Increasing folds $=$ mean relative amount (experimental group)/mean relative amount (control group).

The extra- and intracellular HBV DNA levels in the core particles were detected by real-time PCR analysis as previously described [16]. The culture supernatant and the cytoplasmic lysate of the transfected cells were digested with DNase I to remove plasmid DNA. Subsequently, HBV DNA from the core particles was extracted from $100 \mu \mathrm{L}$ of supernatant or cytoplasmic lysate using a High Pure viral nucleic acid extraction kit (Qiagen) and quantified using the LightCycler-based real-time fluorescence quantitative PCR system $[18,19]$. The amount of extra- and intracellular core particles extracted from each plate of transfected cells was normalized using the expression of Renilla luciferase in the cytoplasmic lysate as an internal control. The decreased $\log _{10}$ value of HBV DNA level $=\left[\right.$ mean $\log _{10}$ value $($ control group) - mean $\log _{10}$ value (experimental group)].

\section{Determination of hepatitis $B$ surface antigen ( $\mathrm{HBsAg})$}

Extracellular HBsAg in the culture supernatant was detected with a commercial assay kit (Abbott Diagnostics). The percentage change $=[$ mean $($ control group $)-$ mean (experiment group)]/mean (control group).

\section{Statistics}

Results are reported as means \pm standard deviation (SD). Differences among groups were tested for significance by one-way analysis of variance (ANOVA). Values of $P<0.05$ were considered significant. All statistical calculations were performed with SPSS10.5 program.

\section{Results}

1. Influence of $\mathrm{HBC}$ protein with hot-spot mutations on the MxA mRNA level and gene promoter activity in Huh7 cells First, we compared the different effect of the HBc proteins (WT and mutated) on the MxA mRNA level in Huh7 cells respectively. Compared to control cells transfected with pCMV-Tag1, MxA mRNA expression induced by IFN- $\alpha$ in Huh7 cells transfected with WT and mutated $\mathrm{HBc}$ proteins (L60V, I97L and S87G) was decreased to $69.1 \%, 69.1 \%, 21.9 \%$ and $73.1 \%$, respectively (Figure 1A), suggesting the L60V and S87G mutated proteins have a similar effect to WT $(p>0.05)$.

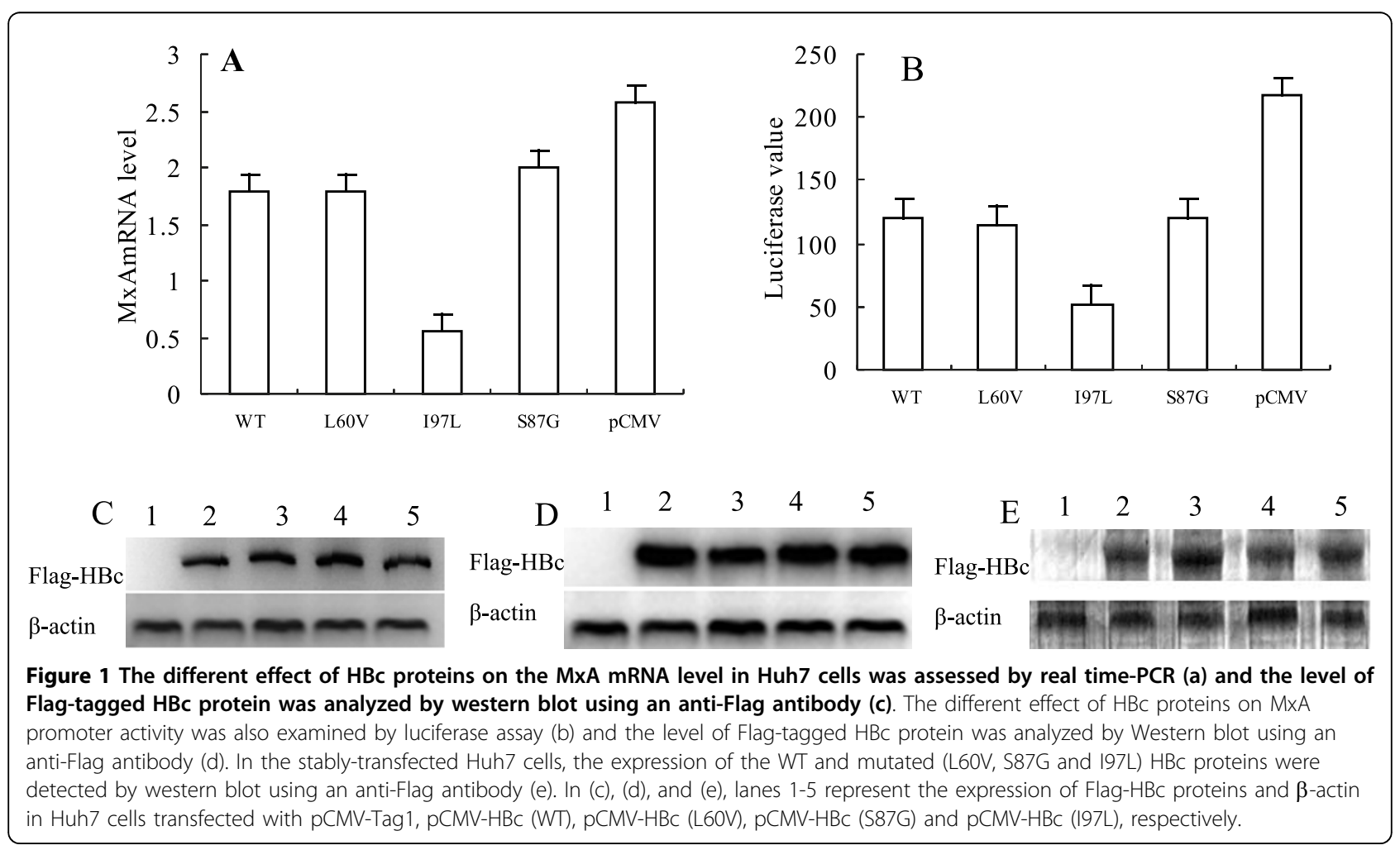


In contrast, I97L HBc protein remarkedly decreased the level of MxA mRNA compared to WT $(p<0.01)$. Subsequently, we investigated the different effects on the promoter activity of MxA gene by the $\mathrm{HBc}$ proteins (WT and mutated). As described in Figure 1B, MxA gene promoter activity in Huh7 cells was influenced to different degrees by the $\mathrm{HBc}$ proteins (WT and mutated). Our data showed that the promoter activity of MxA gene in the Huh7 cells transfected with WT and mutated $\mathrm{HBc}$ proteins (L60V, I97L and S87G) was decreased to $55.2 \%, 53.3 \%, 24.1 \%$ and $55.9 \%$, respectively (Figure 1B) compared to the control cells transfected with pCMV-Tag1. This result showed that I97L HBc protein could remarkably decrease the MxA promoter activity induced by IFN- $\alpha$ compared to the WT or other two mutated HBc proteins (L60V or S87G) $(p<0.01)$. It was consistent with the result on mRNA expression. The expression level of Flag-tagged $\mathrm{HBc}$ protein was analyzed by western blot using an anti-Flag antibody and the results showed no apparent differences in expression of the tagged fusion proteins (Figure 1C,D and $1 \mathrm{E})$.

\section{2. $\mathrm{HBC}$ and its three hot-spot mutants do not affect the inhibition of HBV replication by IFN- $\alpha$}

In order to evaluate whether the inhibition of $\mathrm{MxA}$ transcription by $\mathrm{HBc}$ protein could decrease the inhibitory effect on HBV replication induced by IFN- $\alpha$, pU19-1.24HBV was co-transfected with Renilla luciferase vector into Huh7 cells stably expressing WT HBc protein, L60V, S87G and I97L mutated proteins, respectively. The level of Flag-tagged $\mathrm{HBc}$ protein was analyzed by western blot using an anti-Flag antibody (Figure 1E). The MxA mRNA level was detected by real-time PCR. In the stable expression cells after addition of IFN- $\alpha$, the MxA mRNA levels in the WT, L60V, I97L, S87G and pCMV vector only transfected cells were increased by 5.91 -fold, 5.33-fold, 1.80-fold, 8.30fold, and 20-fold respectively compared to those without IFN- $\alpha$ (Figure 2A). The extracellular HBsAg in these groups of cells was decreased to $58.2 \%, 54.5 \%, 57.4 \%$, $50.6 \%$ and $54.4 \%$, respectively $(p>0.05)$ (Figure $2 \mathrm{~B}$ ) compared to groups non-treated by IFN- $\alpha$ (Figure $2 \mathrm{~B}$ ); the decreased values of extracellular HBV DNA level were $2.00,2.01,1.99,1.93$ and $2.06 \log _{10}$, respectively $(p>0.05)$ (Figure 2C); and the decreased values of the intracellular HBV DNA were 1.03, 1.03, 1.01, 0.94 and $1.09 \log _{10}$, respectively $(p>0.05)$ (Figure $\left.2 \mathrm{D}\right)$. We observed the similar result that WT and hot-spot mutant HBcs inhibitted MxA mRNA expression in stably transfected cells and mutant 197L has most dramatic effect (Fig 2A), this is consistent with our finding in transiently transfected cells (Fig 1). Our data also demonstrated that there is no significant change of
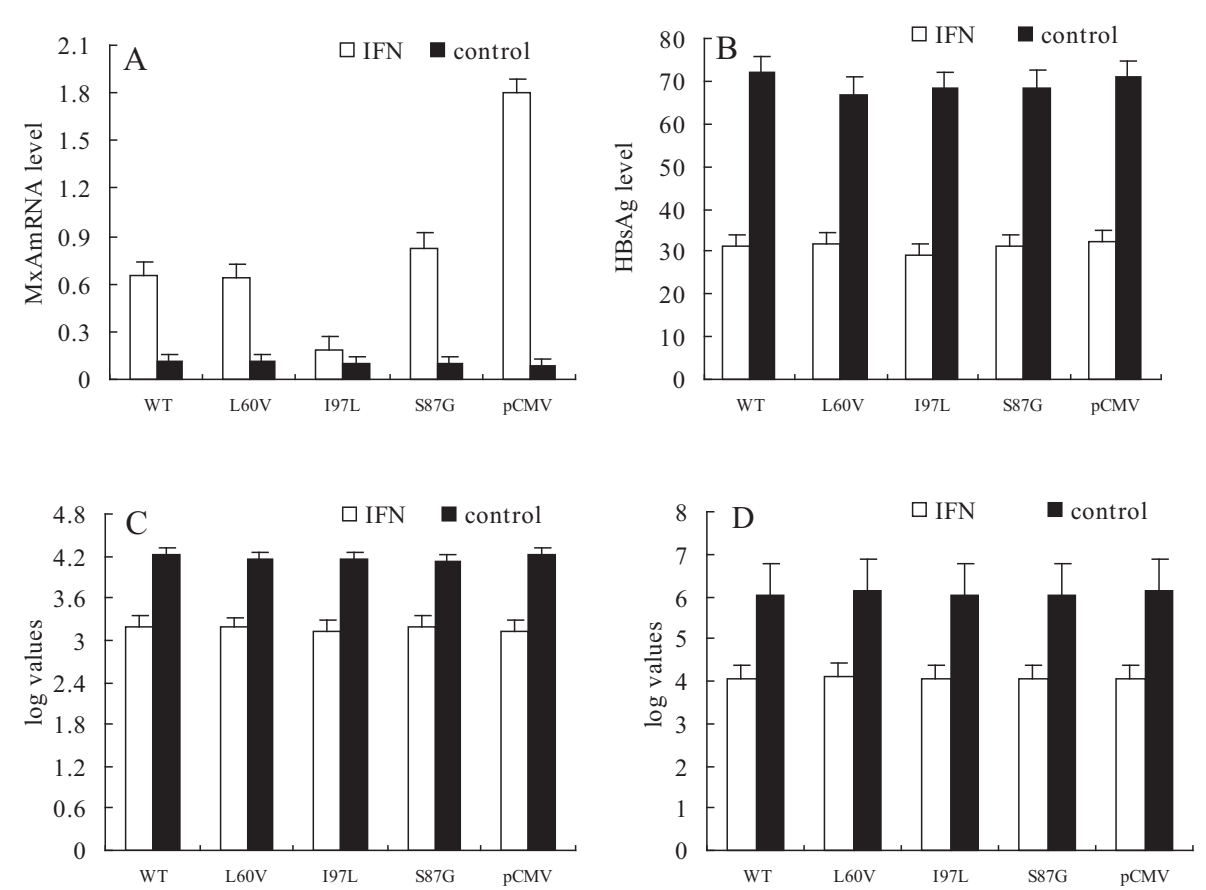

Figure 2 pU19-1.24HBV was co-transfected with Renilla luciferase vector into Huh7 cells stably expressing wild-type HBc protein, L60V, S87G or 197L mutated proteins, as well as into control cells stably transfected with empty vector. MxA mRNA levels in these groups of cells were then detected by real time-PCR (a); the extracellular HBsAg level was assessed by abbot analysis (b) and the extra- and intracellular HBV DNA level (c and d respectively) was measured by real-time PCR. 
HBsAg level, extracellular or intracellular HBV DNA after IFN- $\alpha$ induction among cells stably expressing WT, hot-spot mutant $\mathrm{HBc}$ or expressing no $\mathrm{HBc}$, suggesting that neither WT HBc nor its three hot-spot mutants interfere the inhibitory effect on HBV replication caused by IFN- $\alpha$.

\section{Discussion}

IFN- $\alpha$ has been widely used in the treatment of CHB. The antiviral action of IFN- $\alpha$ is mediated by the induction and activation of at least three IFN-inducible proteins: 2,5-oligoadenylate synthetase (OAS), p68 protein kinase (PK) and the MxA protein [21]. Several in vivo studies have shown a lack of activation of the IFN system in patients with acute or chronic hepatitis B $[10,11,21]$. Recent in vitro studies have suggested that viral factors of HBV might play a direct role in the resistance to the antiviral action of endogenous or exogenous IFN, and multiple reports have demonstrated direct inhibition of the IFN-induced MxA protein expression by the $\mathrm{HBc}$ protein, through interaction with the MxA gene promoter $[9,11,12]$. Different replicative characteristics and clinical significance of full-length HBV strains carrying core protein hot-spot mutations (L60V, S87G and 197L) has also been reported [4-9,22]. However, it is still unknown whether $\mathrm{HBc}$ protein with these hot-spot mutations also has the inhibitory effects on IFN-induced MxA gene expression that are observed with WT. In our study, we showed that L60V and S87G HBc protein decreased the MxA mRNA level induced by IFN- $\alpha$ in Huh7 cells to a similar degree as WT. Moreover, compared with WT and the other two mutants (L60V, S87G), I97L HBc protein remarkably decreased the MxA mRNA level. Consistently, our data also showed that WT, L60V, S87G and I97L HBc protein downregulated the promoter activity of the MxA gene induced by IFN- $\alpha$. In particular, I97L HBc protein remarkably inhibited the MxA promoter activity compared to L60V, S87G or WT. Our result demonstrated in vitro that the HBc hot-spot mutants also inhibited the mRNA level induced by IFN- $\alpha$ and I97L showed significantly more inhibitory effect than that of WT. These results indicated that $\mathrm{HBc}$ protein with certain hot-spot mutations might have a different influence on MxA transcription to that of WT. From this study, we suppose that the mutated $\mathrm{HBc}$ proteins might down-regulate MxA gene transcription by inhibiting promoter activity induced by IFN- $\alpha$ in a similar way to that reported for WT HBc protein[18], but the special inhibitory role of I97L HBc on MxA transcription in Huh7 cells need to be further studied.

Many evidences indicated that the IFN-inducible MxA protein played a key role in the antiviral action of IFN- $\alpha$ against various RNA viruses [13-15]. Recently, Gordien et al reported that MxA was able to inhibit HBV replication by inhibiting the nucleocytoplasmic export of viral mRNAs $[16,17]$. Another study in HBV and HBV/ MxA transgenic mice showed that MxA expression was sufficient to moderately downregulate the expression of viral proteins and reduce the synthesis of HBV DNA, without affecting the steady-state levels of HBV RNAs [23]. However, although several studies (including the present study) have demonstrated the inhibitory effect on transcription and expression of the MxA gene by $\mathrm{HBc}$ protein, it is still necessary to clarify whether this inhibitory effect of $\mathrm{HBc}$ protein on MxA gene transcription can influence the antiviral action of IFN- $\alpha$ on HBV replication. Our data showed that, in Huh7 cells stably expressing WT HBc protein or mutated proteins (L60V, S87G and I97L), IFN- $\alpha$ inhibited the extra- and intracellular levels of HBV DNA, and HBsAg secretion to a similar degree to that in the cells transfected with control plasmids. This suggests that the inhibitory effect on MxA gene transcription by WT or mutated $\mathrm{HBc}$ protein may have no impact on inhibition of HBV replication by IFN- $\alpha$. In fact, Rang et al demonstrated that IFN- $\alpha$ could also suppress HBV replication in MxA-deficient HEp2 cells, indicating that MxA might be not essential for this activity [24]. Frese et al also showed that IFN- $\alpha$ inhibited hepatitis $C$ virus subgenomic RNA replication via an MxA-independent pathway [25]. Therefore, we consider that IFN- $\alpha$ may inhibit HBV replication independently of the MxA pathway in Huh7 cells.

\section{Conclusion}

In conclusion, our data show that $\mathrm{HBc}$ protein with hotspot mutations (L60V, S87G and I97L) can also inhibit MxA gene expression and different mutations have different effect in Huh7 cells; compared to WT HBc protein and the other two mutated proteins (L60V or S87G), HBc protein with the I97L mutation plays an especial role in suppressing transcription and promoter activity of the MxA gene induced by IFN- $\alpha$. Moreover, our results reveal that the inhibition of MxA gene transcription by WT HBc or its hot-spot mutants has no impact on inhibition of HBV replication by IFN- $\alpha$, indicating MxA may be independent of inhibition of HBV DNA replication by IFN- $\alpha$. The clinical significance of the inhibitory effect on MxA gene transcription by $\mathrm{HBc}$ protein requires further study.

\section{Acknowledgements}

This work was supported by two grants from Shenzhen scientific Research Program of the People's Republic of China (NO. 200801020 and NO.

200902174) and also supported by a grant from Ministry of Education of China (NO. 200901121). We thank Wensheng Luo, university of Maryland school of medicine, for critical appraisal of the manuscript. 


\section{Author details}

'Department of Infectious Diseases, the Affiliated Shenzhen Nanshan Hospital of Guangdong Medical College, Shenzhen, China. ${ }^{2}$ Department of of Infectious Diseases, the Affiliated DongHu Hospital of Guangdong Medical College, Shenzhen, China.

\section{Authors' contributions}

$D Q$ and $Z Z$ conceived the study and DQ wrote the paper. $Y Z, H Z, Z F, Y J$ participated in the laboratory studies. All authors read and approved the final manuscript.

\section{Competing interests}

The authors declare that they have no competing interests.

Received: 23 June 2010 Accepted: 20 October 2010

Published: 20 October 2010

\section{References}

1. Mohamadkhani A, Jazii FR, Poustchi H, Nouraein O, Abbasi S, Sotoudeh M, Montazeri $\mathrm{G}$ : The role of mutations in core protein of hepatitis $B$ virus in liver fibrosis. Virol J 2009, 6:209.

2. Mohamadkhani A, Sotoudeh M, Bowden S, Poustchi H, Jazii FR, Sayehmiri K, Malekzadeh R: Downregulation of HLA class II molecules by G1896A precore mutation in chronic hepatitis B virus infection. Viral Immunol 2009, 22:295-300.

3. Sendi H, Mehrab-Mohseni M, Shahraz S, Norder H, Alavian SM, Noorinayer B, Zali MR, Pumpens P, Bonkovsky HL, Magnius LO: CTL escape mutations of core protein are more frequent in strains of $\mathrm{HBeAg}$ negative patients with low levels of HBV DNA. J Clin Virol 2009, 46:259-264.

4. Liu Z, Luo K, He H, Hou J: Hot-spot mutations in hepatitis B virus core gene: eliciting or evading immune clearance? Journ Viral Hepat 2005, 12:146-153.

5. Liu L, Wang YM, Lan L, Li JG, Deng GH, Xia J, Zhao XL, Zhang XQ: Study of the quasispecies dynamics of serum hepatitis $B$ virus in a patient with acute exacerbations of chronic hepatitis B. Zhonghua Gan Zang Bing Za Zhi 2006, 14(1):29-32, (in chinese with english abstract).

6. Kang HY, Lee S, Park SG, Yu J, Kim Y, Jung G: Phosphorylation of hepatitis B virus Cp at Ser87 facilitates core assembly. Biochem J 2006, 398(2):311-317.

7. Yuan $\Pi$, Shih $C:$ A frequent naturally occurring mutation (P130T) of human hepatitis $B$ virus core antigen is compensatory for immature secretion phenotype of another frequent variant (197L). J Virol 2000, 74:4929-4932.

8. Suk FM, Lin MH, Newman M, Pan S, Chen SH, Liu JD, Shih C: Replication advantage and host factor-independent phenotypes attributable to a common naturally occurring capsid mutation (197L) in human hepatitis B virus. J Virol 2002, 76:12069-12077.

9. Pogam SL, Yuan TT, Sahu GK, Chatterjee S, Shih C: Low level secretion of human hepatitis B virus caused by two independent, naturally occurring mutations (P5T and L60V) in the capsid protein. J Virol 2000, 74:9099-9105.

10. Whitten TM, Quets AT, Schloemer RH: Identification of the hepatitis B virus factor that inhibits expression of the beta interferon gene. J Virol $1991,65: 4699-4704$

11. Scaglioni PP, Melegari M, Wands JR: Posttranscriptional regulation of hepatitis B virus replication by the precore protein. J Virol 1997. 71:345-353.

12. Rosmorduc $\mathrm{O}$, Sirma $H$, Soussan $\mathrm{P}$, Gordien $\mathrm{E}$, Lebon $\mathrm{P}$, Horisberger $\mathrm{M}$, Brechot C, Kremsdorf D: Inhibition of interferon-inducible MxA protein expression by hepatitis B virus capsid protein. J Gen Virol 1999, 80:1253-1262.

13. Krejcová D, Procházková J, Kubala L, Pacherník J: Modulation of cell proliferation and differentiation of human lung carcinoma cells by the interferon-alpha. Gen Physiol Biophys 2009, 28(3):294-301.

14. Haller O, Staeheli P, Kochs G: Interferon-induced Mx proteins in antiviral host defense. Biochimie 2007, 89:812-818.

15. Haller $\mathrm{O}$, Frese $\mathrm{M}$, Kochs $\mathrm{G}: \mathrm{Mx}$ proteins: mediators of innate resistance to RNA viruses. Rev Sci Tech 1998, 17:220-230.
16. Yu ZJ, Wang ZH, Chen JJ, Li H, Lin ZZ, Zhang F, Zhou YP, Hou J: GTPase activity is not essential for the interferon-inducible MxA protein to inhibit the replication of hepatitis B virus. Arch Virol 2008, 153:1677-1684.

17. Gordien E, Rosmorduc O, Peltekian C, Garreau F, Brechot C, Kremsdorf D: Inhibition of hepatitis $B$ virus replication by the interferon-inducible MxA protein. J Virol 2001, 75:2684-2691.

18. Fernández M, Quiroga JA, Carreño V: Hepatitis B virus downregulates the human interferon-inducible MxA promoter through direct interaction of precore/core proteins. J Gen Virol 2003, 84(8):2073-2082.

19. Deng $Q$, Zeng $W, Y u Z$ : Signal peptide of Arabinosidase enhances secretion of interferon-alpha2b protein by Bifidobacteria longum. Arch Microbiol 2009, 191(9):681-686.

20. Airò P, Ghidini C, Zanotti C, Scarsi M, Cattaneo R, Caimi L, Imberti L: Upregulation of myxovirus-resistance protein A: a possible marker of type I interferon induction in systemic sclerosis. J Rheumatol 2008, 35(11):2192-2200.

21. Tangkijvanich $P$, Komolmit $P$, Mahachai $V$, Sa-nguanmoo $P$, Theamboonlers A, Poovorawan $Y$ : Low pretreatment serum HBsAg level and viral mutations as predictors of response to PEG-interferon alpha- $2 \mathrm{~b}$ therapy in chronic hepatitis B. J Clin Virol 2009, 46(2):117-123.

22. Schormann W, Kraft A, Ponsel D, Bruss V: Hepatitis B virus particle formation in the absence of pregenomic RNA and reverse transcriptase. J Virol 2006, 80(8):4187-4190.

23. Peltekian C, Gordien E, Garreau F, Meas-Yedid V, Soussan P, Willams V, Chaix ML, Olivo-Marin JC, Bréchot C, Kremsdorf D: Human MxA protein participates to the interferon-related inhibition of hepatitis $B$ virus replication in female transgenic mice. J Hepatol 2005, 43(6):965-972.

24. Rang A, Bruns M, Heise T, Will H: Antiviral Activity of Interferon-a against Hepatitis B Virus Can Be Studied in Non-hepatic Cells and Is Independent of MxA. J Biol chem 2002, 277:7645-7647.

25. Frese M, Pietschmann T, Moradpour D, Haller O, Bartenschlager R: Interferon-a inhibits hepatitis $C$ virus subgenomic RNA replication by an MxA-independent pathway. J Gen Virol 2001, 82:723-733.

doi:10.1186/1743-422X-7-278

Cite this article as: Zhijian et al: Hepatitis B virus core protein with hotspot mutations inhibit MxA gene transcription but has no effect on inhibition of virus replication by interferon $\alpha$. Virology Journal 2010 7:278.

\section{Submit your next manuscript to BioMed Central and take full advantage of:}

- Convenient online submission

- Thorough peer review

- No space constraints or color figure charges

- Immediate publication on acceptance

- Inclusion in PubMed, CAS, Scopus and Google Scholar

- Research which is freely available for redistribution

Submit your manuscript at www.biomedcentral.com/submit
C Biomed Central 